\title{
Finite-size effects of a left-handed material slab on the image quality
}

\author{
Long Chen ${ }^{1}$, Sailing $\mathrm{He}^{1,2, *}$ and Linfang Shen ${ }^{1}$ \\ ${ }^{1}$ Centre for Optical and Electromagnetic Research, \\ State Key Laboratory of Modern Optical Instrumentation, \\ Zhejiang University, Hangzhou Yuquan 310027, P. R. China \\ ${ }^{2}$ Laboratory of Photonics and Microwave Engineering, \\ Department of Microelectronics and Information Technology, \\ Royal Institute of Technology, S-16440 Kista, Sweden
}

\begin{abstract}
The characteristics of an imaging system formed by a left-handed material (LHM) slab of finite length are studied, and the influence of the finite length of the slab on the image quality is analyzed. Unusual phenomena such as surface bright spots and negative energy stream at the image side are observed and explained as the cavity effects of surface plasmons excited by the evanescent components of the incident field. For a thin LHM slab, the cavity effects are found rather sensitive to the length of the slab, and the bright spots on the bottom surface of the slab may stretch to the image plane and degrade the image quality.
\end{abstract}

Keywords: left-handed material, finite size, surface plasmon, cavity

PACS numbers: 78.20.Ci, 42.30.Wb, 73.20.Mf 
Recently, a new type of composite material (also called left-handed material (LHM) since the electric field, the magnetic field and the wave vector of an electromagnetic plane wave propagating in such a material obey the left-hand rule) which exhibits simultaneously negative effective permittivity and permeability over a certain frequency band has attracted a great attention 1, 2, 3, 4]. The extraordinary electromagnetic properties of LHM include reverse Doppler shift, backward-directed Cherenkov radiation cone and inverse Snell effect, which were first analyzed theoretically by Veselago [1].

It has been noticed recently that an infinitely-extended LHM slab can focus not only the propagating waves from the object, but also the evanescent waves corresponding to the subwavelength structure of the object. Therefore, theoretically such a LHM slab can reconstruct the original object and achieve a perfect resolution under an ideal case (i.e., the so-called perfect lens) [3]. While the inherent material loss will greatly suppress the amplification of evanescent waves and make the perfect image impossible [5], subwavelength imaging is still achievable for a thin LHM slab [6]. However, in a realistic imaging system the length of the LHM slab must also be finite, particularly for applications such as micro-detectors and micro-imaging devices where the device size is required to be as small as possible. In this letter, we study the effects of the finite size of the LHM slab to the imaging quality. A finite-difference time-domain (FDTD) method [7] is used in the numerical simulation.

The two-dimensional (2D) imaging system we consider here is a planar LHM slab (surrounded by vacuum) with a finite length of $L$ and a thickness of $d$. The slab is located in the region of $(-L / 2<x<L / 2,0<z<d)$, and a point source located at $(x=0, z=-u)$ is used to generate the object for the imaging system (see the inset of Fig. 1(a)). Here we consider only the E-polarization where $\mathbf{E}$ is directed in the $y$ direction. For matched material parameters and $u<d$, the field will be focused at $z=u$ inside the slab and $z=2 d-u$ outside the slab [3]. Here for simplicity we set $u=0.5 d$ in all our imaging simulation. To avoid the field singularity of the point source, the object plane is selected to be slightly $(0.05 \lambda)$ below the point source, and the image plane is shifted correspondingly. The computational domain is bounded by perfect-matched layers (PMLs) and a FDTD method of scattered-field/total-field version is adopted [7]. To avoid the divergence (occurred when the permittivity and permeability are negative) as the time marches in the FDTD simulation, the following Drude's dispersion model [8] for the permittivity and permeability of the LHM 
slab is used,

$$
\varepsilon(\omega)=\varepsilon_{0}\left(1-\frac{\omega_{p e}^{2}}{\omega^{2}}\right), \quad \mu(\omega)=\mu_{0}\left(1-\frac{\omega_{p m}^{2}}{\omega^{2}}\right)
$$

The permittivity and permeability will take negative values for frequencies below $\omega_{p e}$ and $\omega_{p m}$, respectively. Here we assume $\omega_{p e}=\omega_{p m}$ and the material parameters are matched (i.e., $\varepsilon\left(\omega_{0}\right) / \varepsilon_{0}=\mu\left(\omega_{0}\right) / \mu_{0}=-1$; as assumed for a perfect lens) at frequency $\omega_{0}=\omega_{p e} / \sqrt{2}$. To minimize the frequency extension, the time-dependence of the point source is set as $\exp \left(i \omega_{0} t\right) f(t)$, where $f(t)$ is a step function that reaches 1 smoothly in a time duration of $30 T_{0}$ (here the period $T_{0}=2 \pi / \omega_{0}$ ). The grid size of the discretization is $0.01 \lambda$. After enough time steps, the field evolution becomes stable and the stable field is taken as the field at frequency $\omega_{0}$ (the accuracy has been verified by taking the Fourier transform of the time sequence of the field to extract the field at frequency $\omega_{0}$ and thus the dispersion effect is negligible for our monochromatic incidence).

Unusual Phenomena: Interesting phenomena can be observed clearly from Fig. 1(a) for the distributions of the normalized field intensity and $z$ component of the energy stream $\left(S_{z}\right)$ on the image plane. Here we choose $L=8 \lambda$ and $d=0.2 \lambda$. Unlike the ideal imaging for an infinitely-extended LHM slab, next to the central peak (the desired image) the image for a LHM slab of finite length has additional peaks with considerable magnitudes (even exceeding the magnitude of the central peak in some cases). More surprisingly, near the central peak the energy stream $S_{z}$ has large negative values (reaching about $-20 \%$ after normalization for this example). The negative $S_{z}$ on the image plane seems counter-intuitive since there is no scatterer below the slab and the normal energy stream there should simply flow downward (i.e., positive $S_{z}$ ) from the slab. The corresponding 2D distribution of the field intensity is shown in Fig. 1(b). Clearly one sees many bright spots (nearly equi-distanced) distributed along each surface of the LHM slab. Although the field decreases exponentially away from these surface spots, they still stretch to the image plane due to their large magnitudes and consequently damage the image. The two additional peaks in Fig. 1(a) on the image plane are the extension of the two brightest spots centered on the bottom surface of the slab (see Fig. 1(b)). We have increased $d$ (up to $3 \lambda$ ) and varied $L$ (from $1.5 \lambda$ to $9 \lambda$ ) and these unusual phenomena are still observed.

Cavity Effects: In the imaging system of infinitely extended LHM slab with matched material parameters, incident evanescent wave will experience amplification inside the slab 
and thus have strong intensity around the exit (i.e., bottom) interface of the slab (see e.g. 3]). This wave can be conceived as a localized field around this single interface and is exponentially decreasing both into the slab and below the slab. Therefore, the simple term "surface plasmon" (commonly used for a surface-localized wave in metal-related electromagnetism) is "borrowed" to describe this evanescent wave with large field amplitude near the interface. For a point source located above the slab, the field intensity on the upper or bottom surface has a simple profile with only one central peak. Neither the additional surface bright spots nor negative energy stream can be observed for such an infinitely-extended LHM slab. However, when the slab has a finite length, each excited surface plasmon can be roughly conceived as travelling along the bottom surface and encountering a side-end (i.e., the left or right boundary) of the slab. The main part of the energy should be reflected, with the remaining part running across the corner or coupling to radiation. The original and the multiply reflected waves are superimposed to form a standing wave profile. Therefore, the side-ends act as reflecting walls and the finite slab behaves like an one-dimensional cavity for the surface plasmons. This lateral cavity effect leads to the distribution of nearly equi-distanced bright spots (due to the nodal structure of the standing wave) along the exit interface of the LHM slab. Meanwhile, the energy stream $S_{z}$ along the surface of the slab has an oscillating behavior (with a period similar to that of the surface bright spots) and takes negative values at some positions, as shown in Fig. 1(c). Due to the continuity of the normal stream $S_{z}$ on the bottom surface, these negative streams extend to the image plane and cause negative values at the image side. Note that the meaning of "surface plasmon" used here differs from that of "surface polariton" (or "slab plasmon polariton"), which usually refers to the coupled surface waves on both interfaces, i.e., symmetrical or anti-symmetrical eigen modes of the slab. Such eigen modes do not exist for a LHM slab with an infinite length and matched material parameters (see e.g. [6, 9]).

Here we show that these bright spots and negative energy streams result from the resonance of surface plasmons, which are excited only by the evanescent components of the incident wave. Filters for the spatial spectrum are employed to extract the propagating or evanescent components of the incident field. The incident fields are first Fourier transformed with respect to $x$. A window function is applied to these spectra and the modified spectra are then transformed back to the physical space through the inverse Fourier transform. Therefore, a low-pass window function with the upper truncation $k_{x}=k_{0}$ will extract the 
propagating components of the incident field, while a high-pass window function with the lower truncation $k_{x}=k_{0}$ will extract the evanescent components. For graphic clarity we consider here a slab with $L=2 \lambda$ and $d=2 u=0.2 \lambda$. Note that the length of this slab is not too short since $k_{x}$ has a large value (corresponding to a small period of oscillation in $x$ direction. See e.g., there are about nine nodes over the slab length of $2 \lambda$ in Fig. 2(a) below). The 2D field intensity distributions contributed by the full spectrum, the propagating parts and the evanescent parts of the incident field are shown in Figs. 2(a-c), respectively. The field intensity contributed by the propagating components (Fig. 2(b)) is rather simple, with no reflection at the upper and bottom interfaces (as expected), since under present matched material parameters the propagating components should experience simple negative refraction at the upper and bottom interfaces, and the ends of the finite slab causes only some negligible scattering. The small side-lops are due to the limited wavenumbers for the propagating parts $\left(k_{x}<k_{0}\right)$. However, the field intensity contributed by the evanescent components (Fig. 2(c)) has a similar distribution of surface bright spots (only slight difference in their relative magnitudes) as compared with Fig. 2(a) for the case of full spectrum. We have also compared the energy streams along the image plane for these cases. The stream $S_{z}$ has a simple profile and is non-negative everywhere when only the propagating components are included, while oscillating behavior and negative values are observed when the evanescent components are included.

We can also numerically simulate the resonant behavior of a single evanescent wave for our imaging system. The 2D distributions of the field intensity and energy stream for the case of $L=2 \lambda, d=0.2 \lambda$ and $k_{x}=2 k_{0}$ are shown in Figs. 2(d) and 2(e), respectively. Equi-distanced bright spots along the surface with a period of $\pi / k_{x}=0.25 \lambda$ are clearly seen in Fig. 2(d), while in Fig. 2(e) the energy stream $S_{z}$ exhibits an oscillating property with alternating positive and negative values along the $x$ direction. As $L$ varies, we do observe that the resonance strength (measured by the maximal field intensity $|E|^{2}$ along the bottom surface) oscillates with $L$ with a period around $0.25 \lambda$ and has multiple peak values, resembling the resonant behavior of a simple one-dimensional resonator.

In addition to the lateral resonance caused by the reflection at the side-ends as analyzed above, the bright spots along the upper surface (see e.g. Fig. 2(d)) suggest a coupling mechanism between the upper and bottom surfaces through the slab ends, and can be explained here by a dynamic procedure. For a LHM slab of infinite length with matched 
material parameters, the amplification of a single evanescent wave should produce strong field intensity only around the bottom surface. However, for a LHM slab of finite length, along the bottom surface the amplified evanescent wave diffracts near the side-ends and the corners and couples some part of energy back into the slab. Like an additional evanescent wave incident from the other side of the slab, the diffracted wave will also experience an amplification inside the slab and lead to an enhanced field along the upper surface. Similar diffractions happen recursively around both surfaces until a stable field distribution with bright spots on both surfaces is established, introducing a cavity effect also along the $z$ direction. The field evolution in our FDTD simulation verifies this dynamic procedure, and under some cases (e.g. $L=2.2 \lambda, k_{x}=2.8 k_{0}$ ) the field strength along the upper interface is much stronger than that along the bottom interface.

Here we present some numerical analysis of the two-dimensional cavity effects (i.e. the dependence of the resonant strength on the wave-vector $k_{x}$, the length $L$ and the thickness $d$ ). Fig. 3(a) shows the resonant strength of different $k_{x}$ for $d=0.2 \lambda$ and $L=2.0 \lambda$ (diamonds), $2.2 \lambda$ (circles) and $2.5 \lambda$ (squares), respectively. Here to balance the amplification inside the slab for a fair comparison between different $k_{x}$, we keep the source-slab distance $u=d$. As mentioned above, the resonant strength depends greatly on $L$, and it also has a selective effect on $k_{x}$ with multiple resonant peaks (marked by arrow, other peaks beyond the range of this figure are observed and relatively have much weaker amplitude). Additionally, these cases share the same peak positions (see e.g. $k_{x} \approx 2.25 k_{0}$ ), which suggests that the resonant $k_{x}$ (which excites the strongest resonance along the slab surfaces) shall be related to the coupling between the two slab surfaces (i.e., the other dimension $d$ of the proposed cavity), and the slab length $L$ mainly influences the amplitude. As shown in Fig. 3(b) for $L=2.0 \lambda$, the resonant peak positions shift monotonously from $k_{x} \approx 2.6 k_{0}$ to $2.25 k_{o}$ and $1.65 k_{0}$ for $d$ from $0.15 \lambda$ (diamonds) to $0.2 \lambda$ (circles) and $0.3 \lambda$ (squares), and their corresponding peak values decrease for about one order for $d$ from $0.15 \lambda$ to $0.3 \lambda$.

An incident field generated by a point source contains many evanescent waves. Due to the sensitivity of the resonant strength of each evanescent component to length $L$, the overall field for the case of a point source is complex and depends greatly on $L$. A simple comparison for the field intensity profiles along the bottom surface and the corresponding images are shown in Fig. 4(a) and (b) for two slightly different values of $L$ (i.e., $L=2 \lambda$ and $2.2 \lambda$ ), respectively. Here the thickness is still kept as $d=0.2 \lambda$. Much brighter surface 
spots exist for $L=2 \lambda$, and along the image plane the two unwanted large peaks around $x= \pm 0.23 \lambda$ are the extension of the two distinct surface spots centered on the bottom surface of the slab. Contrarily, the case of $L=2.2 \lambda$ gives a flattened central spot and weaker field in the side-lop region, and thus the image quality is better as compared with that for $L=2 \lambda$. As we increase $L$ further, the field intensity profiles are found to exhibit a quasi-periodic behavior. For $L=2.4 \lambda$, the image quality becomes poor again with two unwanted peaks greatly exceeding the central peak. The additional surface spots still exist and degrade the image quality for $L$ up to $9 \lambda$, and the image quality varies between the best cases of clear image e.g. $L=8.2 \lambda$ with the full width at half maximum (FWHM) about $0.24 \lambda$ (the FWHM of the object is about $0.22 \lambda$ ) and the worst cases (e.g. $L=2.4 \lambda$ ) that the expected point image is greatly exceeded by the additional resonant peaks (by more than 130\%). However, the distance between these resonant peaks has greatly decreased from about $0.23 \lambda$ to $0.11 \lambda$ when we increase $L$ from $2 \lambda$ to $9 \lambda$. For a sufficiently long slab, the resonant spectrum shall tend to become flat and these lateral nodal structures are expected to partially cancel each other except for the central peak (i.e., the desired image). Thus, the influence of the cavity effects on the image decreases gradually and the field intensity distribution gradually transits to the simple shape for a LHM slab of infinite length. For a thicker slab, the resonance becomes weaker and more flat (see Fig. 3(b)), and its influence on the image quality decreases because of its evanescent characteristics along the $z$ direction and the larger distance between the image plane and the bottom surface of the slab. It is also less sensitive to the length of the slab. In our simulation for $d=\lambda$, along the image plane the intensity of these additional peaks are always less than $30 \%$ of that of the central peak (the desired image) for $L>7 \lambda$, and the negative streams are less than $-2 \%$, producing a clear point image with FWHM about $0.3 \lambda$. Similarly, as we have verified, the material loss of the LHM slab (if included) also damps the excitation of surface plasmons and their cavity effects.

In conclusion, the imaging system formed by a LHM slab of finite length has been analyzed through a FDTD method. The cavity effects of surface plasmons excited by the evanescent components of the incident field have been studied and used to explain the observed bright spots along the surfaces of the LHM slab and counter-intuitive negative values of the normal component of the energy stream at the image side of the slab. The extended bright spots on the bottom surface of the LHM slab may stretch to the image plane and degrade the image 
quality. It has been shown that both the length and thickness of the LHM slab greatly influences the cavity effects of surface plasmons and consequently the image quality.

Acknowledgments: The partial support of National Natural Science Foundation of China (under a key project grant; grant number 90101024) is gratefully acknowledged. The authors are also grateful to the referees for their valuable comments.

* Electronic Address: sailing@tet.kth.se

[1] V. G. Veselago, Sov. Phys. Usp. 10, 509 (1968).

[2] R. A. Shelby et al., Science 292, 77(2001).

[3] J. B. Pendry, Phys. Rev. Lett. 85, 3966 (2000).

[4] D. R. Smith et al., Phys. Rev. Lett. 85, 2933 (2000); C. G. Parazzoli et al., ibid. 90, 107401 (2003); A. A. Houck et al., ibid. 90, 137401 (2003); J. Pacheco et al., ibid. 89, 257401 (2003);

A. A. Zharov et al., ibid. 91, 037401 (2003).

[5] N. Garcia et al., Phys. Rev. Lett. 88, 207403 (2002).

[6] D. R. Smith et al., Appl. Phys. Lett. 82, 1506 (2003).

[7] A. Taflove, Computational Electrodynamics: The Finite-Difference Time-Domain Method, Artech House (1995).

[8] S. A. Cummer, Appl. Phys. Lett. 82, 1503 (2003).

[9] R. Ruppin, J. Phys.:Condens. Matter 13, 1811 (2001). 


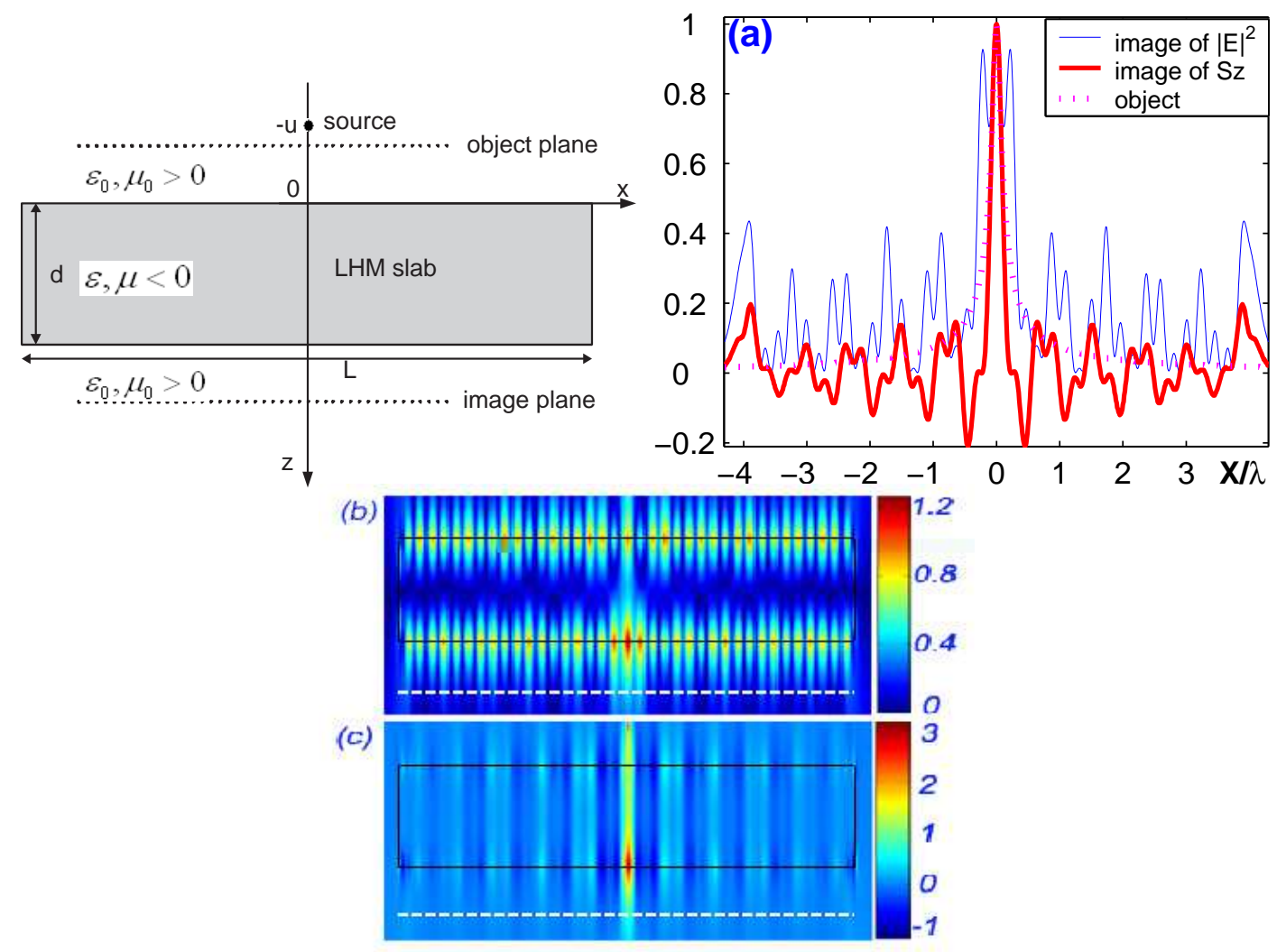

FIG. 1: (a) Normalized distribution of field intensity (thin line) and the energy stream $S_{z}$ (thick line) along the image plane for $L=8 \lambda$ and $d=0.2 \lambda$; here the normalized field intensity of the object is plotted (dotted line) for comparison. Inset: the geometry of the imaging system formed by a LHM slab of finite length. The corresponding 2D distribution of (b) $|E|^{2}$ and (c) $S_{z}$. Here the LHM slab ively.

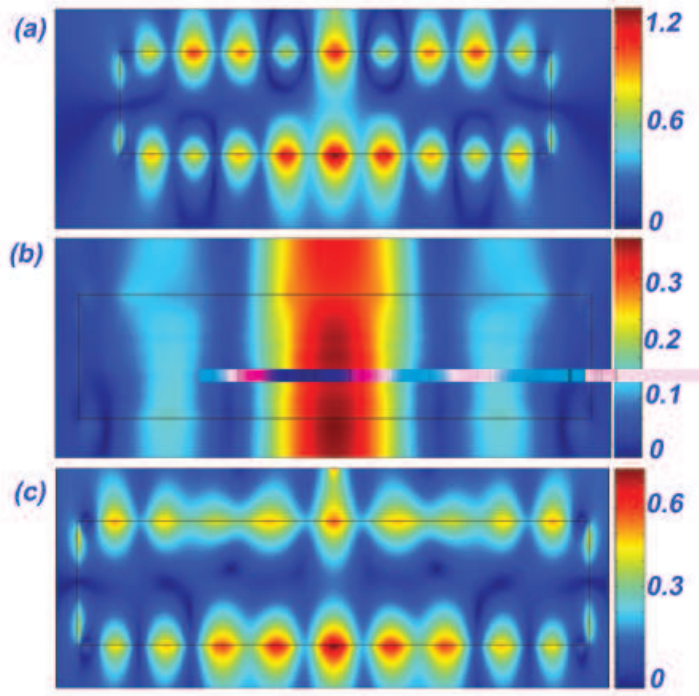




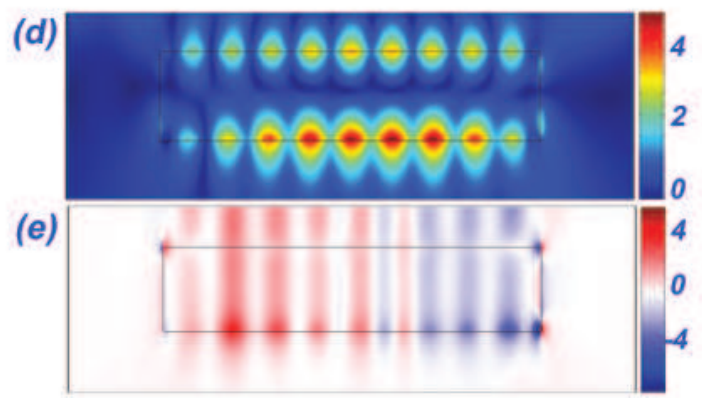

FIG. 2: (a-c) 2D distribution of the field intensity after applying a filter to the spatial spectrum of the incident field generated by a point source: (a) with the full spectrum (b) with only the propagating parts (c) with only the evanescent parts of the spatial spectrum. (d) and (e) give the $2 \mathrm{D}$ distributions of the field intensity and $S_{z}$, respectively, when the resonance is excited by a single evanescent wave with $k_{x}=2 k_{0}$. Here $L=2 \lambda$ and $d=2 u=0.2 \lambda$.
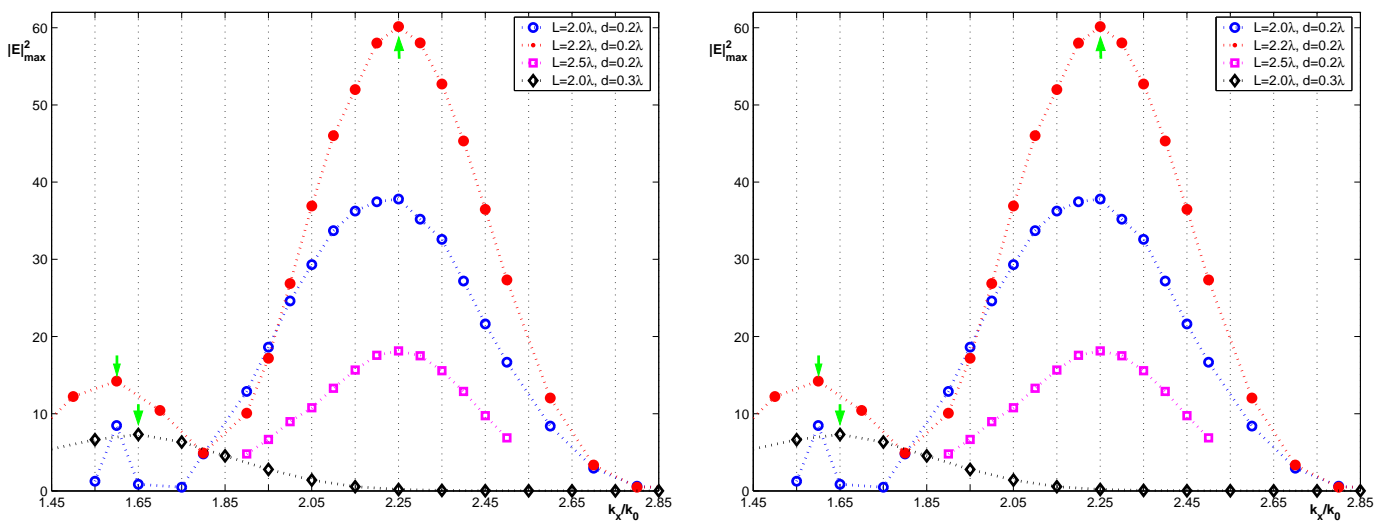

FIG. 3: Dependence of the resonant strength on $k_{x}$ : (a) for $d=0.2 \lambda$ and $L=2.0 \lambda$ (diamonds), $2.2 \lambda$ (circles) and $2.5 \lambda$ (squares), respectively; (b) for $L=2.0 \lambda$ and $d=0.15 \lambda$ (diamonds), $0.2 \lambda$ (circles) and $0.3 \lambda$ (squares), respectively. 

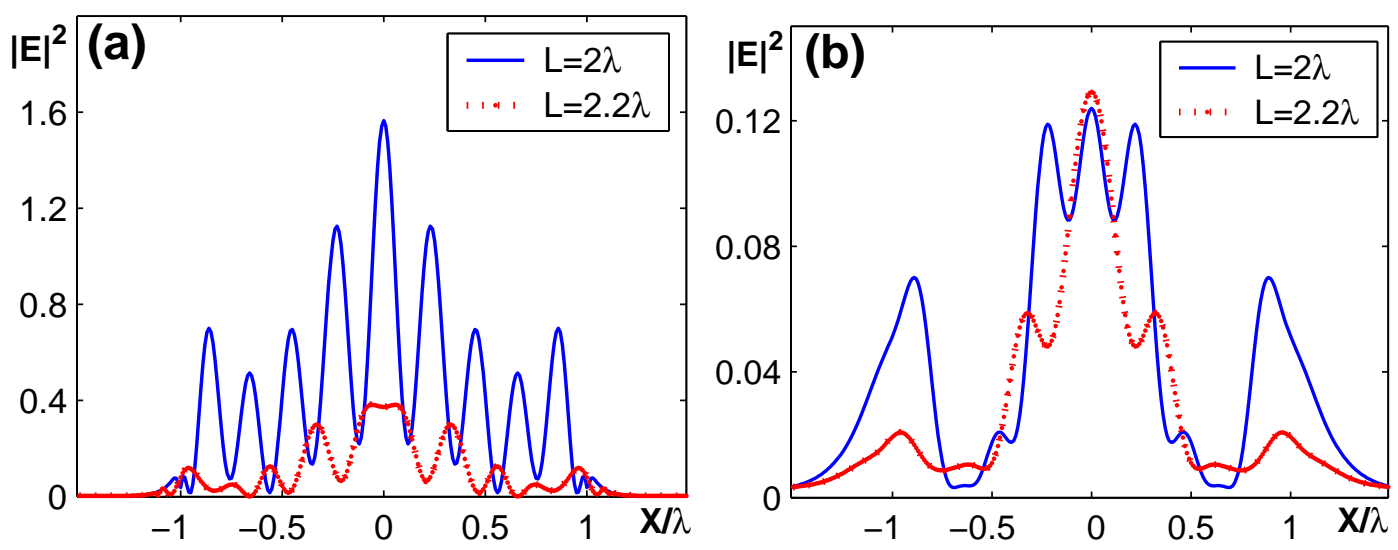

FIG. 4: Field intensity profiles (a) along the bottom surface of the LHM slab and (b) along the image plane, when the incident field is generated by a point source. Here $d=2 u=0.2 \lambda$. 